\title{
Predictors of community-associated Staphylococcus aureus, methicillin-resistant and methicillin-susceptible Staphylococcus aureus skin and soft tissue infections in primary-care settings
}

\author{
G. C. LEE ${ }^{1,2 *}$, R. G. HALL $2 \mathrm{ND}^{3,4}$, N. K. BOYD ${ }^{1,2}$, S. D. DALLAS ${ }^{5}$, L. C. DU ${ }^{6}$, \\ L. B. TREVIÑO ${ }^{6}$, C. RETZLOFF ${ }^{6}$, S. B. TREVIÑO ${ }^{6}$, K. A. LAWSON ${ }^{1}$, \\ J. P. WILSON ${ }^{1}$, R. J. OLSEN ${ }^{7}, Y$. WANG ${ }^{8}$ AND C. R. FREI ${ }^{1,2}$ \\ ${ }^{1}$ College of Pharmacy, The University of Texas at Austin, Austin, TX, USA; ${ }^{2}$ Pharmacotherapy Education \\ and Research Center, School of Medicine, The University of Texas Health Science Center, San Antonio, TX, \\ USA; ${ }^{3}$ Texas Tech University Health Sciences Center, School of Pharmacy, Dallas, TX, USA; ${ }^{4}$ Dose \\ Optimization and Outcomes Research (DOOR) Program, Dallas, TX, USA; ${ }^{5}$ Department of Clinical \\ Laboratory Sciences, School of Health Professions, University of Texas Health Science Center, San Antonio, TX, \\ USA; ${ }^{6}$ South Texas Ambulatory Research Network, The University of Texas Health Science Center, San \\ Antonio, TX, USA; ${ }^{7}$ Department of Clinical Pathology and Genomic Medicine, Methodist Research Institute, \\ Houston, TX, USA; ${ }^{8}$ Department of Biology, The University of Texas San Antonio, San Antonio, TX, USA
}

Received 18 March 2016; Final revision 7 July 2016; Accepted 12 July 2016;

first published online 4 August 2016

\section{SUMMARY}

Skin and soft tissue infections (SSTIs) due to Staphylococcus aureus have become increasingly common in the outpatient setting; however, risk factors for differentiating methicillin-resistant S. aureus (MRSA) and methicillin-susceptible S. aureus (MSSA) SSTIs are needed to better inform antibiotic treatment decisions. We performed a case-case-control study within 14 primarycare clinics in South Texas from 2007 to 2015. Overall, 325 patients [S. aureus SSTI cases (case group 1, $n=175$ ); MRSA SSTI cases (case group 2, $n=115$ ); MSSA SSTI cases (case group 3, $n=60$ ); uninfected control group (control, $n=150$ )] were evaluated. Each case group was compared to the control group, and then qualitatively contrasted to identify unique risk factors associated with $S$. aureus, MRSA, and MSSA SSTIs. Overall, prior SSTIs [adjusted odds ratio (aOR) 7·60, 95\% confidence interval (CI) 3·31-17·45], male gender (aOR 1·74, 95\% CI $1 \cdot 06-2 \cdot 85$ ), and absence of healthcare occupation status (aOR 0.14, 95\% CI 0.03-0.68) were independently associated with $S$. aureus SSTIs. The only unique risk factor for communityassociated (CA)-MRSA SSTIs was a high body weight ( $\geqslant 110 \mathrm{~kg}$ ) (aOR 2.03, 95\% CI 1.01-4.09).

Key words: Epidemiology, soft tissue infections, Staphylococcus aureus.

\section{INTRODUCTION}

The incidence of outpatient and emergency-department visits for skin and soft tissue infections (SSTIs)

\footnotetext{
* Author for correspondence: G. C. Lee, PharmD, PhD, BCPS, Pharmacotherapy Education and Research Center, School of Medicine, The University of Texas Health Science Center at San Antonio, 7703 Floyd Curl Drive, MC 6220, San Antonio, TX 78229-3900, USA.

(Email: leeg3@uthscsa.edu)
}

has substantially increased with the emergence of community-associated methicillin-resistant $S$. aureus (CA-MRSA) [1]. In the United States, about $80-90 \%$ of SSTIs are due to S. aureus [2]. Moreover, there is significant geographical diversity in the prevalence of CA-MRSA and methicillin-susceptible $S$. aureus (MSSA) strains in the community setting. Understanding the risk factors for CA-MRSA can help direct public health interventions and guide clinical management; however, the ability of traditional and newly identified 
risk factors to distinguish CA-MRSA infections from CA-MSSA infections has been widely inconsistent and population dependent. Several studies have investigated risk factors for CA-MRSA SSTIs, but these have been primarily based among inpatients or posthospitalized patients, paediatric populations, and in the context of outbreak investigations [3-6]. Studies focusing on the adult community population are limited [7] and prior studies have been compromised by a lack of an uninfected control group. Such a comparison is important in identifying distinct risk factors for the development of community-associated $S$. aureus SSTIs and to distinguish unique risk factors for MRSA from MSSA.

We have recently described the prevalence and treatment characteristics of CA-MRSA SSTIs in South Texas in the primary-care setting $[8,9]$. The objective of this study was to investigate risk factors for CA-MRSA SSTI and CA-MSSA SSTI compared to uninfected controls.

\section{METHODS}

We performed this investigation among a welldescribed cohort of patients with SSTIs in the primary-care setting; details of this cohort have been described previously $[8,9]$. The study was conducted in collaboration with 14 clinics within the South Texas Ambulatory Research Network (STARNet), a practice-based research network composed of 108 urban, suburban, and rural primary-care clinics distributed throughout the South Texas region, from 2007 to 2015. Patients were eligible for study enrolment if they provided informed consent, were aged $\geqslant 18$ years, and presented to one of the participating clinics with a SSTI. Control patients were enrolled through a subsequent prospective study within the same participating clinics and were eligible for study enrolment if they met the consent and age criteria above but did not have a SSTI or other infection from January 2015 to May 2015. Healthcare providers collected patient information.

A nested case-case-control study was conducted to identify risk factors associated with MRSA and MSSA SSTIs compared to controls [10]. Four study groups were defined: case group 1 comprised patients with either MRSA or MSSA SSTIs to represent CA$S$. aureus as one risk group $(n=175)$. Case group 2 comprised patients with MRSA SSTIs $(n=115)$. Case group 3 comprised patients with MSSA SSTIs $(n=60)$. The uninfected control group (control) comprised patients who presented to these clinics without an SSTI, and represented the 'at risk' community population $(n=150)$. This method allowed us to assess the effect difference of infections due to methicillin-susceptible strains, those with methicillinresistant strains, and those shared by both, compared to the same controls.

For microbiological analyses, samples were plated onto blood agar plates (TSA with $5 \%$ sheep blood; Fisher Scientific, USA) and incubated at $35-37^{\circ} \mathrm{C}$ for $24 \mathrm{~h}$, then subcultured to MRSA selective agar (MRSASelect chromogenic agar plates; Bio-Rad Laboratories, USA). Latex agglutination tests (StaphAurex ${ }^{\circledR}$; ThermoFisher Scientific, USA), and phenotypic screening tests (cefoxitin resistance using VITEK 2 AST-GP75 cards, bioMérieux, USA) were used for the isolation and identification of MRSA. Antimicrobial minimum inhibitory concentrations were interpreted according to the Clinical and Laboratory Standards Institute document M100-S24 (2014) [11].

Clinical information collected included patient gender, race (Black, White, Other), ethnicity (Hispanic, Non-Hispanic), past medical history (e.g. diabetes, peripheral vascular disease, chronic non-infectious skin disorder, HIV/AIDS, cancer, actively receiving chemotherapy, immunosuppression), healthcarerelated work history, skin infection history, height, weight, infection characteristics (e.g. location, duration, size, deepest tunnel depth, erythema, smell, ulceration, drainage, abscess, satellites), incision and drainage procedures received, and antibiotics prescribed. A weight $\geqslant 110 \mathrm{~kg}$ was used to indicate 'high body weight'. This is consistent with previous literature associating high body weight with antimicrobial dosing outcomes $[12,13]$.

Bivariable analyses were conducted comparing patient demographics, comorbidities, and exposures of each case group to the control group. Categorical variables were evaluated using Pearson's $\chi^{2}$ or Fisher's exact test, and continuous variables were evaluated with Student's $t$ test or Wilcoxon rank sum test. Odds ratios (ORs) and 95\% confidence intervals (CIs) are reported. For multivariable analyses, three backward stepwise logistic regression models were conducted for each case group reporting adjusted ORs (aORs) and $95 \%$ CIs. Variables were entered into the models if: (1) their $P$ values were $<0 \cdot 10$ in the bivariable between-group analyses; and (2) more than $5 \%$ of the study cohort had the presence of the variables. $P \leqslant 0.05$ was used to determine statistical 
significance. Finally, we qualitatively considered the independent predictors for each comparison to identify only those uniquely associated with MRSA or MSSA SSTIs. SPSS v. 22.0 (IBM Corp, USA) was used for all statistical comparisons.

\section{RESULTS}

Overall, 175 patients with $S$. aureus SSTIs were evaluated: 115 patients with MRSA-positive cultures and 60 patients with MSSA-positive cultures. One hundred and fifty patients with no SSTI were enrolled as controls. Table 1 depicts the bivariable analyses and Table 2 depicts the multivariable analyses.

Case-control study 1: S. aureus SSTI cases vs. controls

In bivariable analysis, patients with $S$. aureus SSTIs were more likely to report a prior SSTI in the last 90 days (OR 5·84, 95\% CI 2.75-12.40), more likely to weigh $\geqslant 110 \mathrm{~kg}$ (OR 1.92, 95\% CI 1.06-3.46), more likely to be male (OR $1 \cdot 70,95 \%$ CI $1 \cdot 09-2 \cdot 65$ ), and less likely to have a healthcare occupation (OR $0 \cdot 11,95 \%$ CI $0 \cdot 30-0 \cdot 51)$ compared to controls. In multivariable analysis, prior SSTIs (aOR 7.60, 95\% CI 3.31-17.45), male gender (aOR 1.74, 95\% CI 1.06-2.85), and absence of healthcare occupation status (aOR 0.14, 95\% CI 0.03-0.68) were independently associated with $S$. aureus SSTIs. A high body weight was not statistically significant (aOR $1 \cdot 58,95 \%$ CI $0 \cdot 82-3 \cdot 06)$.

\section{Case-control study 2: MRSA SSTI cases vs. controls}

In bivariable analysis, most patient demographics including age, male gender, race, and ethnicity were similar between MRSA SSTI cases and controls. Furthermore, there was no difference in the frequency of prior antimicrobial exposures between the two groups. Patients with MRSA SSTIs were more likely to report a prior SSTI in the last 90 days (OR 7.53, $95 \%$ CI $3 \cdot 54-16 \cdot 42$ ), more likely to weigh $\geqslant 110 \mathrm{~kg}$ (OR 2.54, 95\% CI 1.34 4.81), and less likely to have a healthcare occupation (OR $0 \cdot 16,95 \%$ CI $0 \cdot 04$ $0.71)$ compared to controls. In multivariable analysis, a history of prior SSTI in the last 90 days (aOR 7.98, $95 \%$ CI $3 \cdot 37-18 \cdot 88)$, high body weight $(\geqslant 110 \mathrm{~kg}$ ) (aOR 2.03, 95\% CI 1.01-4.09), and healthcare occupation (aOR $0 \cdot 15,95 \%$ CI $0 \cdot 03-0 \cdot 74$ ) were independently associated with MRSA SSTIs.

\section{Case-control study 3: MSSA SSTI cases vs. controls}

In bivariable analyses, patients with MSSA SSTIs were significantly more likely to report a prior SSTI (OR $3 \cdot 12,95 \%$ CI $1 \cdot 22-8 \cdot 33$ ), more likely to be male (OR 2.57, 95\% CI 1.39-4.84), and less likely to have a healthcare occupation (OR $0 \cdot 90,95 \%$ CI $0 \cdot 85-0 \cdot 95$ ) compared to controls. In multivariable analysis, a history of prior SSTI in the last 90 days (aOR 4.82, 95\% CI 1.75-13.29), male gender (aOR 2.01, 95\% CI $1 \cdot 03$ to 3.94 ), and healthcare occupation (aOR $0 \cdot 17,95 \%$ CI $0.02-0.88)$ were independently associated with MSSA SSTIs.

\section{Qualitative comparison of models}

Male gender, non-healthcare occupation, and prior SSTIs were predictors for $S$. aureus as an overall entity. After qualitative comparison of risk factors for MRSA SSTIs and MSSA SSTIs compared to controls, a high body weight $(\geqslant 110 \mathrm{~kg})$ was a unique risk factor for MRSA SSTI cases, while male gender was the only unique risk factor for MSSA cases.

\section{DISCUSSION}

Over the past 10 years, ambulatory care visits for SSTIs have increased [14]. The worldwide emergence of CA-MRSA strains has made the management of $S$. aureus SSTIs extremely complicated and challenging. A clinical approach to the management of $S$. aureus SSTIs is to identify risk factors that can predict and possibly differentiate infections with MRSA and MSSA.

In this study, we found that a high body weight $(\geqslant 110 \mathrm{~kg})$ was associated with CA-MRSA-related SSTIs. Obesity has been a known risk factor for SSTIs; however, the association between obesity or high body weight and CA-MRSA-related SSTIs has not been widely recognized. An outbreak investigation among New York football players identified a higher BMI and sharing towels were independently associated with MRSA infections [5]. More recently, Khawcharoenporn et al. identified obesity to be an independent risk factor for MRSA SSTIs compared to patients with SSTIs due to other bacterial aetiologies [15]. One hypothesis is that the higher risk MRSA SSTIs in this population may represent a manifestation of virulence factors such as the arginine catabolic mobile element (ACME) associated with USA300 CA-MRSA strains. Specifically, the spe $G$ gene within 
Table 1. Bivariable analyses of risk factors associated with community-associated methicillin-resistant $\mathbf{S}$. aureus, methicillin-susceptible $\mathbf{S}$. aureus, and $\mathbf{S}$. aureus skin and soft tissue infections compared to non-infected controls

\begin{tabular}{|c|c|c|c|c|c|c|c|}
\hline Characteristic & $\begin{array}{l}\text { MRSA } \\
(n=115)\end{array}$ & $\begin{array}{l}\text { MSSA } \\
(n=60)\end{array}$ & $\begin{array}{l}\text { S. aureus } \\
(n=175)\end{array}$ & $\begin{array}{l}\text { Controls } \\
(n=150)\end{array}$ & $\begin{array}{l}\text { MRSA } v s . \\
\text { controls } \\
P\end{array}$ & $\begin{array}{l}\text { MSSA vs. } \\
\text { controls } \\
P\end{array}$ & $\begin{array}{l}\text { S. aureus vs. } \\
\text { controls } \\
P\end{array}$ \\
\hline Mean age, years $( \pm$ s.D. $)$ & $40( \pm 13)$ & $42( \pm 14)$ & $41( \pm 13)$ & $43( \pm 14)$ & $0 \cdot 27$ & $0 \cdot 70$ & $0 \cdot 30$ \\
\hline Male & $53(46 \%)$ & $36(60 \%)$ & $89(51 \%)$ & $58(39 \%)$ & $0 \cdot 21$ & $<0 \cdot 01^{*}$ & $0 \cdot 02 *$ \\
\hline \multicolumn{8}{|l|}{ Race/ethnicity } \\
\hline Black & $8(7 \%)$ & $1(2 \%)$ & $9(5 \%)$ & $12(8 \%)$ & $0 \cdot 77$ & $0 \cdot 12 \dagger$ & $0 \cdot 37$ \\
\hline Hispanic & $86(75 \%)$ & $47(78 \%)$ & $133(76 \%)$ & $119(79 \%)$ & $0 \cdot 45$ & $0 \cdot 78$ & $0 \cdot 59$ \\
\hline Diabetes & $25(22 \%)$ & $17(28 \%)$ & $44(25 \%)$ & $34(23 \%)$ & $0 \cdot 89$ & $0 \cdot 17$ & $0 \cdot 60$ \\
\hline Weight $>110 \mathrm{~kg}$ & $29(25 \%)$ & $11(18 \%)$ & $37(21 \%)$ & $20(13 \%)$ & $<0 \cdot 01^{*}$ & $0 \cdot 82$ & $0 \cdot 04 *$ \\
\hline Chronic non-infectious skin disorder & 0 & $1(2 \%)$ & $1(1 \%)$ & $3(2 \%)$ & $1 \cdot 00 \dagger$ & $0 \cdot 39 \dagger$ & $1 \cdot 00 \dagger$ \\
\hline HIV & 0 & 0 & 0 & $1(1 \%)$ & $1 \cdot 00 \dagger$ & $1 \cdot 00 \dagger$ & $1 \cdot 00 \dagger$ \\
\hline Cancer & 0 & 0 & 0 & $5(3 \%)$ & $0 \cdot 58 \dagger$ & $1 \cdot 00 \dagger$ & $0 \cdot 33 \dagger$ \\
\hline Provides healthcare to others & $2(2 \%)$ & $1(2 \%)$ & $3(2 \%)$ & $15(10 \%)$ & $<0.01 * \dagger$ & $<0.01 * \dagger$ & $<0.01 * \dagger$ \\
\hline Prior SSTI & $37(32 \%)$ & $10(17 \%)$ & $47(27 \%)$ & $9(6 \%)$ & $<0 \cdot 01^{*}$ & $0 \cdot 01^{*}$ & $<0 \cdot 01 *$ \\
\hline Prior antibiotics within 90 days & $18(16 \%)$ & $9(15 \%)$ & $27(15 \%)$ & $26(17 \%)$ & $0 \cdot 74$ & $0 \cdot 72$ & $0 \cdot 76$ \\
\hline
\end{tabular}

MRSA, Methicillin-resistant S. aureus; MSSA, methicillin-susceptible $S$. aureus; s.D., standard deviation; HIV, human immunodeficiency virus; SSTI, skin and soft tissue infection.

No patients had history of peripheral vascular disease or chemotherapy.

* Statistically significant.

$\dagger$ Fisher's exact test was used. 
Table 2. Multivariable analyses of risk factors for community-associated $\mathbf{S}$. aureus, methicillin-resistant $\mathbf{S}$. aureus, and methicillin-susceptible $\mathrm{S}$. aureus skin and soft tissue infections compared to non-infected controls

\begin{tabular}{|c|c|c|c|c|c|c|}
\hline \multirow[b]{2}{*}{ Variables } & \multicolumn{2}{|c|}{ S. aureus vs. controls } & \multicolumn{2}{|l|}{ MRSA vs. controls } & \multicolumn{2}{|l|}{ MSSA vs. controls } \\
\hline & aOR $(95 \% \mathrm{CI})$ & $P$ & $\mathrm{aOR}(95 \% \mathrm{CI})$ & $P$ & aOR $(95 \% \mathrm{CI})$ & $P$ \\
\hline Male & $1.74(1.06-2 \cdot 85)$ & $0 \cdot 04$ & & & $2 \cdot 01(1.03-3.94)$ & 0.04 \\
\hline Healthcare provider & $0 \cdot 14(0 \cdot 03-0 \cdot 68)$ & $<0 \cdot 01$ & $0 \cdot 15(0 \cdot 03-0 \cdot 74)$ & $0 \cdot 02$ & $0 \cdot 17(0 \cdot 02-0 \cdot 88)$ & $0 \cdot 02$ \\
\hline Weight $>110 \mathrm{~kg}$ & $1.58(0.82-3 \cdot 06)$ & $0 \cdot 17$ & $2.03(1.01-4.09)$ & 0.04 & & \\
\hline History of prior SSTI & $7 \cdot 60(3 \cdot 31-17 \cdot 45)$ & $<0 \cdot 01$ & $7 \cdot 98(3 \cdot 37-18 \cdot 88)$ & $<0 \cdot 01$ & $4 \cdot 82(1 \cdot 75-13 \cdot 29)$ & $<0 \cdot 01$ \\
\hline
\end{tabular}

MRSA, Methicillin resistant S. aureus; MSSA, methicillin susceptible S. aureus; SSTI, skin and soft tissue infection; aOR, adjusted odds ratio; CI, confidence interval.

the ACME locus was found to be associated with increased resistance to polyamines that are produced on the skin, which are toxic to other $S$. aureus strains $[16,17]$. This fitness advantage of ACME-containing MRSA strains, coupled with the changes that compromise skin structure in patients with high body fat, may cause a specific pathogen-host interaction in this population [18, 19]. This increased risk for MRSA SSTIs, and not MSSA SSTIs, in patients with high body weight merits further investigation, particularly because obese patients have been associated with increased risk for complications and recurrences of infection [19].

Male gender was a distinct independent risk factor for MSSA SSTIs. This finding contrasts with other studies that showed no significant gender predominance nor identified male gender to be a risk factor for MRSA infections [20, 21]. The majority of studies describing the relationship of men and MRSA infections were conducted in institutionalized settings, men who have sex with men populations, sports teams, and military recruits. Conversely, studies have shown that men are at higher risk for MSSA bacteraemia and MSSA colonization [22-24]. Colonization has been shown to be an important risk factor preceding infections; this may, in part, reflect a higher incidence of MSSA SSTIs in men. However, we did not account for colonization in this study. Other possible reasons why gender may play a role in developing MSSA or MRSA infections include different health-seeking behaviours, social behaviours (e.g. occupation, recreational activities), the infecting clones, or hormonal differences. Regardless, these are mostly speculative and further epidemiological investigation is needed.

There are limitations to this study. First, we did not account for social and behavioural risk factors that may be associated with $S$. aureus SSTIs and/or clinical outcomes. Second, we used laboratory diagnosis to identify $S$. aureus cases. Patients presenting with SSTIs with no culture or with culture-negative results may have different characteristics. SSTIs can be caused by a variety of pathogens, including $S$. aureus, streptococci, and Gram-negative bacteria. This study focused on the subset of patients with positive cultures for $S$. aureus. These patients may present differently from the general SSTI population. Further, based on the inclusion criteria, patients without suspected MRSA infections may not have been accounted for. The small sample size limited the ability to identify risks associated with lower exposures. Another limitation is the long time period of the study, during which secular trends of CA-MRSA have been described [25, 26]. Finally, there may be limited generalizability to other regions outside of South Texas. Strengths of this study include the case-case-control design to assess potential epidemiological risk factors for communityassociated $S$. aureus infections compared to uninfected controls and to distinguish between MRSA and MSSA. This methodology allowed us to identify common and unique risk factors that were associated with MRSA or MSSA SSTIs. Finally, this study was based in the primary-care setting, adding important findings to the sparse literature in this growing population.

In summary, in outpatients with $S$. aureus SSTIs in South Texas, we found minimal differences in predictors for MRSA and MSSA SSTIs. Overall, prior SSTIs, male gender, and non-healthcare occupation were predictors for $S$. aureus SSTIs. The only unique risk factor for CA-MRSA SSTIs was a high body weight.

\section{ACKNOWLEDGEMENTS}

The authors thank their South Texas Ambulatory Research Network (STARNet) and Area Health Education Center (AHEC) colleagues, including 
Paula Winkler, who assisted with the administrative aspects of the study.

This study was funded by an investigator-initiated research grant from Pfizer to Dr Frei. Dr Frei was also supported by the U.S. National Institutes of Health in the form of a KL2 Career Development Award (NIH/ NCRR 5KL2 RR025766, PI-Robert Clark).

\section{DECLARATION OF INTEREST}

None.

\section{REFERENCES}

1. Pallin DJ, et al. Increased US emergency department visits for skin and soft tissue infections, and changes in antibiotic choices, during the emergence of communityassociated methicillin-resistant Staphylococcus aureus. Annals of Emergency Medicine 2008; 51: 291-298.

2. Ray GT, Suaya JA, Baxter R. Incidence, microbiology, and patient characteristics of skin and soft-tissue infections in a U.S. population: a retrospective populationbased study. BMC Infectious Diseases 2013; 13: 252.

3. Sattler CA, Mason Jr. EO, Kaplan SL. Prospective comparison of risk factors and demographic and clinical characteristics of community-acquired, methicillinresistant versus methicillin-susceptible Staphylococcus aureus infection in children. Pediatric Infectious Disease Journal 2002; 21: 910-917.

4. Skiest DJ, et al. Prospective comparison of methicillinsusceptible and methicillin-resistant community-associated Staphylococcus aureus infections in hospitalized patients. Journal of Infection 2007; 54: 427-434.

5. Centers for Disease Control and Prevention. Methicillinresistant Staphylococcus aureus among players on a high school football team - New York City, 2007. Morbidity Mortality Weekly Report 2009; 58: 52-55.

6. Popovich KJ, et al. Community-associated methicillinresistant Staphylococcus aureus and HIV: intersecting epidemics. Clinical Infectious Diseases 2010; 50: 979-987.

7. Miller LG, et al. Clinical and epidemiologic characteristics cannot distinguish community-associated methicillin-resistant Staphylococcus aureus infection from methicillin-susceptible $S$. aureus infection: a prospective investigation. Clinical Infectious Diseases 2007; 44: 471-482.

8. Forcade NA, et al. Prevalence, severity, and treatment of community-acquired methicillin-resistant Staphylococcus aureus (CA-MRSA) skin and soft tissue infections in 10 medical clinics in Texas: a South Texas Ambulatory Research Network (STARNet) study. Journal of the American Board of Family Medicine 2011; 24: 543-550.

9. Labreche MJ, et al. Treatment failure and costs in patients with methicillin-resistant Staphylococcus aureus (MRSA) skin and soft tissue infections: a South Texas Ambulatory Research Network (STARNet) study. Journal of the American Board of Family Medicine 2013; 26: 508-517.
10. Kaye KS, et al. The case-case-control study design: addressing the limitations of risk factor studies for antimicrobial resistance. Infection Control and Hospital Epidemiology 2005; 26: 346-351.

11. Clinical Laboratory Standards Institute. Performance standards for antimicrobial susceptibility testing. M100-S24. Wayne, PA: Clinical and Laboratory Standards Institute, 2014.

12. Rubino CM, et al. Oritavancin population pharmacokinetics in healthy subjects and patients with complicated skin and skin structure infections or bacteremia. Antimicrobial Agents and Chemotherapy 2009; 53: 4422-4428.

13. Falagas ME, Karageorgopoulos DE. Adjustment of dosing of antimicrobial agents for bodyweight in adults. Lancet 2010; 375: 248-251.

14. Hersh AL, et al. National trends in ambulatory visits and antibiotic prescribing for skin and soft-tissue infections. Archives of Internal Medicine 2008; 168: 1585-1591.

15. Khawcharoenporn $\mathbf{T}$, et al. Risk factors for communityassociated methicillin-resistant Staphylococcus aureus cellulitis - and the value of recognition. Journal of Hawaii Medicine 2010; 69: 232-236.

16. Diep BA, et $\boldsymbol{a l}$. The arginine catabolic mobile element and staphylococcal chromosomal cassette mec linkage: convergence of virulence and resistance in the USA300 clone of methicillin-resistant Staphylococcus aureus. Journal of Infectious Diseases 2008; 197: 1523-1530.

17. Planet PJ, et al. Emergence of the epidemic methicillinresistant Staphylococcus aureus strain USA300 coincides with horizontal transfer of the arginine catabolic mobile element and speG-mediated adaptations for survival on skin. MBio 2013; 4: e00889-13.

18. Early GJ, Seifried SE. Risk factors for communityassociated Staphylococcus aureus skin infection in children of Maui. Hawaii Journal of Medicine and Public Health 2012; 71: 218-223.

19. Yosipovitch G, DeVore A, Dawn A. Obesity and the skin: skin physiology and skin manifestations of obesity. Journal of American Academy of Dermatology 2007; 56: 901-916; quiz 17-20.

20. Kupfer M, et al. MRSA in a large German university hospital: male gender is a significant risk factor for MRSA acquisition. GMS Krankenhhyg Interdisziplinar 2010; 5: Doc11.

21. Casey JA, et al. A population-based study of the epidemiology and clinical features of methicillin-resistant Staphylococcus aureus infection in Pennsylvania, 20012010. Epidemiology and Infection 2013; 141: 1166-1179.

22. Klevens RM, et al. Invasive methicillin-resistant Staphylococcus aureus infections in the United States. Journal of the American Medical Association 2007; 298: 1763-1771.

23. Laupland KB, Ross T, Gregson DB. Staphylococcus aureus bloodstream infections: risk factors, outcomes, and the influence of methicillin resistance in Calgary, Canada, 2000-2006. Journal of Infectious Diseases 2008; 198: 336-343.

24. Graham 3rd PL, Lin SX, Larson EL. A U.S. populationbased survey of Staphylococcus aureus colonization. Annals of Internal Medicine 2006; 144: 318-325. 
25. Delorenze GN, et al. Trends in annual incidence of methicillin-resistant Staphylococcus aureus (MRSA) infection in HIV-infected and HIV-uninfected patients. Epidemiology and Infection 2013; 141: 2392-2402.
26. Landrum ML, et al. Epidemiology of Staphylococcus aureus blood and skin and soft tissue infections in the US military health system, 2005-2010. Journal of the American Medical Association 2012; 308: 50-59. 